\title{
Hopf Bifurcation Analysis for the Modified Rayleigh Price Model with Time Delay
}

\author{
Yanhui Zhai, Haiyun Bai, Ying Xiong, and Xiaona Ma \\ School of Science, Tianjin Polytechnic University, Tianjin 300387, China \\ Correspondence should be addressed to Yanhui Zhai; y_h_zhai@aliyun.com
}

Received 9 May 2013; Accepted 28 June 2013

Academic Editor: Sakthivel Rathinasamy

Copyright ( 2013 Yanhui Zhai et al. This is an open access article distributed under the Creative Commons Attribution License, which permits unrestricted use, distribution, and reproduction in any medium, provided the original work is properly cited.

\begin{abstract}
This paper mainly modifies and further develops the Reyleigh price model. By modifying the basic Reyleigh model, we can more accurately illustrate the economic phenomena with price varying. First, we research the dynamics of the modified Reyleigh model with time delay. By employing the normal form theory and center manifold theory, we obtain some testable results on these issues. The conclusion confirms that a Hopf bifurcation occurs due to the existence of stability switches when the delay varies. Finally, some numerical simulations are given to illustrate the effectiveness of our results.
\end{abstract}

\section{Introduction}

With the rapid development of economic society. As one of the many important economic problems, the price oscillation has been widely accepted by many people, especially some researchers. Since the price is a main factor of affecting supply and demand, many researchers have been devoted to study the price model. Different researchers may apply different price models to solve the practical problems. In this paper, our research is based on the traditional Reyleigh price model [1]. As for this model, many researchers have widely studied it by using different methods and proposed some new ideals. However, there is only a limited number of analytical works on the model with time delay. Although in [1] Lv and Liu have studied the Reyleigh model with time delay, they consider the situation that supply depends on the price of the past only. In order to finely interpret economic phenomena, our research is based on the fact that supply depends not only on the price of the past but also on the present price. So, the main purpose of this study is to provide an insight into these unexplored aspects of the Reyleigh price model with time delay.

The traditional Reyleigh price model is described by the following two-dimensional autonomous system:

$$
\begin{gathered}
\dot{x}(t)=-y(t)+l\left(\frac{1}{3} a x^{3}(t)+\frac{1}{2} b x^{2}(t)+c x(t)\right), \\
\dot{y}(t)=x(t),
\end{gathered}
$$

where $x(t)$ is the price at time $t, y(t)$ is the amount of supply at time $t$, and $a, b, c, l$ are the constants.

By introducing the time delay, the above system (1) can be transformed into the following form:

$$
\begin{gathered}
\dot{x}(t)=-y(t)+l\left(\frac{1}{3} a x^{3}(t)+\frac{1}{2} b x^{2}(t)+c x(t)\right), \\
\dot{y}(t)=x(t-\tau),
\end{gathered}
$$

where $\tau$ is positive and the other parameters are the same as (1).

In [1], by employing the $\tau-D$ partitioning approach, Lv and Liu have systematically discussed some complex dynamic behaviors of system (2).

Now, based on the economic meaning and the fact discussed at the beginning of the introduction, we modify system (2) as follows:

$$
\begin{gathered}
\dot{x}(t)=-y(t)+l\left(\frac{1}{3} a x^{3}(t)+\frac{1}{2} b x^{2}(t)+c x(t)\right), \\
\dot{y}(t)=\frac{1}{2} d x(t)+\frac{1}{2} k x(t-\tau),
\end{gathered}
$$

where $d, k$ refer to [2]. 


\section{The Stability Analysis}

In this section, we obtain the domain of the stable equilibrium when time delay $\tau$ varies from small to large. And further, by applying the Hopf bifurcation theorem, we give the condition of the Hopf bifurcation.

It is known that, on the one hand, if the equilibrium of system (3) is stable when $\tau=0$ and the characteristic equation of (3) has no purely imaginary roots for any $\tau>0$, it is also stable for any $\tau>0$. On the other hand, if the equilibrium of system (3) is stable when $\tau=0$ and there exist some positive values $\tau$ such that the characteristic equation of (3) has a pair of purely imaginary roots, there exists a domain concerning $\tau$ such that the equilibrium of system (3) is stable in the domain.

Obviously, system (3) has the only equilibrium $(0,0)$. And the linearization of system $(3)$ at $(0,0)$ is

$$
\begin{gathered}
\dot{x}(t)=-y(t)+l c x(t), \\
\dot{y}(t)=\frac{1}{2} d x(t)+\frac{1}{2} k x(t-\tau),
\end{gathered}
$$

whose characteristic equation is

$$
\lambda^{2}-l c \lambda+\frac{1}{2} k e^{-\lambda \tau}+\frac{1}{2} d=0
$$

When the case $\tau=0$, we have the following.

Lemma 1. (i) When $c<0$, the equilibrium $(0,0)$ of system (3) is stable.

(ii) When $c>0$, the equilibrium $(0,0)$ of system (3) is unstable.

(iii) When $c=0$ and $b<0(b>0)$, the equilibrium $(0,0)$ of system (3) is stable (unstable).

The proof is straightforward, and we omit it.

Lemma 2. If $d^{2}<k^{2}$ is satisfied, the characteristic equation (5) has a pair of purely imaginary roots $\pm i \omega_{0}$ when $\tau=\tau_{j}$, where

$$
\begin{aligned}
& \omega_{0}=\left(\frac{-\left(l^{2} c^{2}-d\right)+\sqrt{\left(l^{2} c^{2}-d\right)^{2}-\left(d^{2}-k^{2}\right)}}{2}\right)^{1 / 2}, \\
& \tau_{j}=\frac{\arccos \left(\left(2 \omega_{0}-d\right) / k\right)+2 j \pi}{\omega_{0}}, \quad j=0,1,2, \ldots
\end{aligned}
$$

Proof. Let $i \omega(\omega>0)$ is a root of (5). Then

$$
-\omega^{2}-l c \omega i+\frac{1}{2} d+\frac{1}{2} k(\cos (\omega \tau)-i \sin (\omega \tau))=0 .
$$

The separation of the real and imaginary parts yields

$$
\begin{gathered}
-\omega^{2}+\frac{1}{2} k \cos (\omega \tau)+\frac{1}{2} d=0 \\
-l c \omega-\frac{1}{2} k \sin (\omega \tau)=0
\end{gathered}
$$

which lead to

$$
\omega^{4}-d \omega^{2}+l^{2} c^{2} \omega^{2}+\frac{1}{4} d^{2}-\frac{1}{4} k^{2}=0 .
$$

By solving the second-degree equation (9) concerning $\omega^{2}$, we have

$$
\omega^{2}=\frac{-\left(l^{2} c^{2}-d\right) \pm \sqrt{\left(l^{2} c^{2}-d\right)^{2}-\left(d^{2}-k^{2}\right)}}{2} .
$$

We take

$$
\omega_{0}=\left(\frac{-\left(l^{2} c^{2}-d\right)+\sqrt{\left(l^{2} c^{2}-d\right)^{2}-\left(d^{2}-k^{2}\right)}}{2}\right)^{1 / 2} .
$$

And, hence

$$
\tau_{0}=\frac{\arccos \left(\left(2 \omega_{0}^{2}-d\right) / k\right)}{\omega_{0}} .
$$

By setting $\tau_{j}=\tau_{0}+\left(2 j \pi / \omega_{0}\right), j=0,1,2, \ldots$. Then $\omega_{0}, \tau_{j}$ satisfies the condition of Lemma 2.

The proof is completed.

According to Lemmas 1 and 2 and the assertion at the beginning of this section, we know there must exist some finite interval with regard to $\tau$ in which the equilibrium $(0,0)$ is stable.

Now we investigate how the real part of the roots of (5) varies as $\tau$ varies in a small neighbourhood of $\tau_{j}$.

Assume that $\lambda(\tau)=a(\tau)+i b(\tau)$ is the root of the characteristic equation (5), and it meets $a\left(\tau_{j}\right)=0, b\left(\tau_{j}\right)=$ $\omega_{0}$. By differentiating both sides of (5) with regard to $\tau$ and solving $\lambda^{\prime}(\tau)$, we obtain

$$
\lambda^{\prime}(\tau)=\frac{(1 / 2) k \lambda e^{-\lambda \tau}}{2 \lambda-l c-(1 / 2) k \tau e^{-\lambda \tau}} .
$$

That is,

$$
\lambda^{\prime}\left(\tau_{j}\right)=\frac{(1 / 2) k \omega_{0} i e^{-\omega_{0} i \tau_{j}}}{2 \omega_{0} i-l c-(1 / 2) k \tau_{j} e^{-\omega_{0} i \tau_{j}}} .
$$

We substitute (8) into the above equation and separate the real and imaginary parts, and we have

$$
\operatorname{Re}\left\{\lambda^{\prime}\left(\tau_{j}\right)\right\}=\frac{2 \omega_{0}^{4}+(l c-d) \omega_{0}^{2}}{\left((1 / 2) d \tau_{j}-l c-\omega_{0}^{2} \tau_{j}\right)^{2}+\left(2 \omega_{0}-l c \omega_{0} \tau_{j}\right)^{2}}
$$

When $l c>d$,

$$
\operatorname{Re}\left\{\lambda^{\prime}\left(\tau_{j}\right)\right\}>0 .
$$

Here, we know that the root of (5) crosses the imaginary axis from the left to the right as $\tau$ continuously varies from a number less than $\tau_{j}$ to one greater than $\tau_{j}$. Thus, when $\tau>\tau_{0}$, 
the characteristic equation (5) has at least one root with positive real part. Further, the equilibrium $(0,0)$ is unstable in the interval $\left(\tau_{0},+\infty\right)$.

By applying Lemmas 1 and 2 and condition (*), we have the following results.

Theorem 3. If $c<0, k^{2}>d^{2}$ and $l c>d$ hold, the equilibrium $(0,0)$ of system (3) is asymptotically stable for $\tau \in\left[0, \tau_{0}\right)$ and unstable for $\tau \in\left(\tau_{0},+\infty\right)$. System (3) exhibits the Hopf bifurcation at the equilibrium $(0,0)$ for $\tau=\tau_{j}, j=0,1,2, \ldots$.

\section{Hopf Bifurcation Analysis}

In Section 2, we obtain the conditions under which family periodic solutions bifurcate from the steady state at the critical value of $\tau$. In this section, by applying the normal form and centre manifold theory, we discuss the direction and stability of the bifurcating periodic solutions. Throughout this section, we always assume that system (3) meets the conditions of the Hopf bifurcation.

By time scaling $t \rightarrow t / \tau$, system (3) is transformed into the following form:

$$
\begin{gathered}
\dot{x}(t)=-\tau y(t)+l \tau\left(\frac{1}{3} a x^{3}(t)+\frac{1}{2} b x^{2}(t)+c x(t)\right), \\
\dot{y}(t)=\frac{1}{2} \tau d x(t)+\frac{1}{2} \tau k x(t-1) .
\end{gathered}
$$

It is not difficult to show that system (16) also exhibits the Hopf bifurcation at the equilibrium $(0,0)$ for $\tau=\tau_{j}$ $(j=0,1,2, \ldots)$. Without loss of generality, we only consider the bifurcation parameter $\tau_{0}$. For convenience, by setting $\tau=$ $\tau_{0}+\mu$, system (16) is rewriten as

$$
\begin{gathered}
\dot{x}(t)=-\left(\tau_{0}+\mu\right) y(t)+l\left(\tau_{0}+\mu\right) \\
\times\left(\frac{1}{3} a x^{3}(t)+\frac{1}{2} b x^{2}(t)+c x(t)\right), \\
\dot{y}(t)=\frac{1}{2}\left(\tau_{0}+\mu\right) d x(t)+\frac{1}{2}\left(\tau_{0}+\mu\right) k x(t-1) .
\end{gathered}
$$

Clearly, $\mu=0$ is the Hopf bifurcation value of system (17).

Throughout the following section, $C_{1}=C\left([-1,0] ; R_{+}^{2}\right)$ is a phase space and the superscripts " $T$ " and "*" stand for the transpose and adjoint, respectively.

In $C_{1}$, system (17) can be written as the following equation:

$$
\dot{X}(t)=\left(\tau_{0}+\mu\right) L\left(X_{t}\right)+\left(\tau_{0}+\mu\right) F\left(X_{t}\right),
$$

where $X(t)$ is a vector $\left(x_{1}(t), x_{2}(t)\right), X_{t}=X(t+\theta)$ for $\theta \epsilon$ $[-1,0]$, and $L, F$ are given by

$$
\begin{aligned}
& L(\phi)=\left(\begin{array}{c}
l c \phi_{1}(0)-\phi_{2}(0) \\
\frac{1}{2} d \phi_{1}(0)+\frac{1}{2} k \phi_{1}(-1)
\end{array}\right), \\
& F(\phi)=\left(\begin{array}{c}
\frac{1}{3} a l \phi_{1}^{3}(0)+\frac{1}{2} b l \phi_{1}^{2}(0) \\
0
\end{array}\right),
\end{aligned}
$$

for $\phi=\left(\phi_{1}, \phi_{2}\right) \in C_{1}$.
Now, we consider the abstract functional differential equation [3]:

$$
\dot{u}_{t}=A(\mu) u_{t}+R(\mu) u_{t}
$$

where $u_{t}=u(t+\theta) \in C_{1}$ for $\theta \in[-1,0]$.

The operators $A$ and $R$ are defined as

$$
\begin{gathered}
A(\mu) \phi(\theta)= \begin{cases}\frac{d \phi(\theta)}{d \theta}, & \theta \in[-1,0), \\
\int_{-1}^{0} d(\eta(t, \mu) \phi(t)), & \theta=0,\end{cases} \\
R(\mu) \phi(\theta)= \begin{cases}0, & \theta \in[-1,0), \\
f(\mu, \theta), & \theta=0,\end{cases}
\end{gathered}
$$

where $\int_{-1}^{0} d(\eta(t, \mu) \phi(t))=\left(\tau_{0}+\mu\right) L(\phi)$ (here, $\eta(\theta, \mu)$ is a bounded variation function for $\theta \in[-1,0]), f(\mu, \phi)=\left(\tau_{0}+\right.$ $\mu) F(\phi)$.

Consider the adjoint bilinear form $\langle\cdot, \cdot\rangle$ on $C_{1} \times C_{1}^{*}$ :

$$
\langle\psi, \phi\rangle=\bar{\psi}(0) \phi(0)-\int_{-1}^{0} \int_{0}^{\theta} \bar{\psi}(\xi-\theta) d \eta(\theta) \phi(\xi) d \xi,
$$

where $\eta(\theta)=\eta(\theta, 0)$.

According to the adjoint bilinear form $\langle\cdot, \cdot\rangle$, we can define an adjoint operator $A^{*}(0)$ corresponding to $A(0)$ as the following form:

$$
A^{*} \psi(s)= \begin{cases}-\frac{d \psi(s)}{d s}, & s \in(0,1], \\ \int_{-1}^{0} d\left(\eta^{T}(t, 0) \psi(-t)\right), & s=0 .\end{cases}
$$

To determine the normal form of operator $A$, we need to calculate the eigenvectors $q(\theta)$ and $q^{*}(s)$ of $A$ and $A^{*}$ corresponding to $i \tau_{0} \omega_{0}$ and $-i \tau_{0} \omega_{0}$, respectively.

Proposition 4. Assume that $q(\theta)$ and $q^{*}(s)$ are the eigenvector $q(\theta)$ and $q^{*}(s)$ of $A$ and $A^{*}$ corresponding to $i \tau_{0} \omega_{0}$ and $-i \tau_{0} \omega_{0}$, respectively, satisfying $\left\langle q^{*}, q\right\rangle=1$ and $\left\langle q^{*}, \bar{q}\right\rangle=0$.

Then

$$
\begin{aligned}
& q(\theta)=(\alpha, \beta)^{T} e^{i \omega_{0} \tau_{0} \theta}=\left(1, l c-\omega_{0} i\right)^{T} e^{i \omega_{0} \tau_{0} \theta}, \\
& q^{*}(s)=D\left(\alpha^{*}, \beta^{*}\right) e^{i \omega_{0} \tau_{0} s}=\left(\omega_{0} i, 1\right) e^{i \omega_{0} \tau_{0} s},
\end{aligned}
$$

where $\bar{D}=2 /\left(2 \alpha \alpha^{*}+2 \beta \beta^{*}+k \tau_{0} \alpha \overline{\beta^{*}} e^{-i \omega_{0} \tau_{0}}\right)$.

Proof. Without loss of generality, we just consider the eigenvector $q(\theta)$.

Firstly, when $\theta \in[-1,0)$, by the definition of $A$ and $q(\theta)$, we obtain the form $q(\theta)=(\alpha, \beta)^{T} e^{i \omega_{0} \tau_{0}}$ (here, $\alpha, \beta$ are unknown parameters).

In what follows, notice that $q(0)=(\alpha, \beta)^{T}$ and $A q(0)=$ $\int_{-1}^{0} d(\eta(t, \mu) \phi(t))=i \omega_{0} \tau_{0} q(0)$, and we have $\alpha=1, \beta=l c-\omega_{0} i$.

Finally, by $\left\langle q^{*}, q\right\rangle=1$, we obtain the parameter $\bar{D}$ (refer to $[4,5])$.

The proof is completed. 
As in [6], the bifurcating periodic solutions $x(t, \mu)$ of system (16) are indexed by a small parameter $\varepsilon$. A solution $x(t, \mu(\varepsilon))$ has amplitude $O(\varepsilon)$, period $T(\varepsilon)$, and nonzero Floquet exponent $\beta(\varepsilon)$ with $\beta(0)=0$. Under the present assumptions, $\mu, T$, and $\beta$ have expansions $[7,8]$ :

$$
\begin{gathered}
\mu=\mu_{2} \varepsilon^{2}+\mu_{4} \varepsilon^{4}+\cdots, \\
T=\frac{2 \pi}{\omega}\left(1+T_{2} \varepsilon^{2}+T_{4} \varepsilon^{4}+\cdots\right), \\
\beta=\beta_{2} \varepsilon^{2}+\beta_{4} \varepsilon^{4}+\cdots,
\end{gathered}
$$

where the sign of $\mu_{2}$ determines the directions of the Hopf bifurcations, the sign of $\beta_{2}$ determines the stability of the bifurcation periodic solutions, and $T_{2}$ determines the period of the bifurcating periodic solutions.

The purpose of this section is to compute the coefficients $\mu_{2}, T_{2}, \beta_{2}$ in the above expansions.

Next, we construct the coordinates of the center manifold $C_{0}$ at $\mu=0$. Let

$$
\begin{gathered}
z(t)=\left\langle q^{*}, u_{t}\right\rangle, \\
W(t, \theta)=u_{t}(\theta)-2 \operatorname{Re}\{z(t) q(\theta)\} .
\end{gathered}
$$

On the center manifold $C_{0}$, we have

$$
W(t, \theta)=W(z(t), \overline{z(t)}, \theta)
$$

where

$$
W(z, \bar{z}, \theta)=W_{20}(\theta) \frac{z^{2}}{2}+W_{11}(\theta) z \bar{z}+W_{02} \frac{\bar{z}^{2}}{2}+W_{30} \frac{z^{3}}{6} \cdots
$$

$z$ and $\bar{z}$ are local coordinates for the center manifold $C_{0}$ in the direction of $q$ and $q^{*}$, respectively. Since $\mu=0$, we have

$$
\begin{aligned}
z^{\prime}(t) & =i \tau_{0} \omega_{0} z(t)+\left\langle q^{*}(\theta), f(W+2 \operatorname{Re}\{z(t) q(\theta)\})\right\rangle \\
& =i \tau_{0} \omega_{0} z(t)+\overline{q^{*}(0)} f(W(z, \bar{z}, 0)+2 \operatorname{Re}\{z(t) q(0)\}) \\
& \triangleq i \tau_{0} \omega_{0} z(t)+\overline{q^{*}(0)} f_{0}(z, \bar{z})
\end{aligned}
$$

where

$$
f_{0}(z, \bar{z})=f_{z^{2}} \frac{z^{2}}{2}+f_{\bar{z}^{2}} \frac{\bar{z}^{2}}{2}+f_{z \bar{z}} z \bar{z}+\cdots
$$

We rewrite this as

$$
z^{\prime}(t)=i \tau_{0} \omega_{0} z+g(z, \bar{z})
$$

with

$$
\begin{aligned}
g(z, \bar{z}) & =\overline{q^{*}}(0) f_{0}(z, \bar{z}) \\
& =g_{20} \frac{z^{2}}{2}+g_{11} z \bar{z}+g_{02} \frac{\bar{z}^{2}}{2}+g_{21} \frac{z^{2} \bar{z}}{2}+\cdots
\end{aligned}
$$

Proposition 5. For (31), one has

$$
\begin{aligned}
& \text { (i) } g_{20}=g_{11}=g_{02}=\bar{D} b l \tau_{0} \overline{\alpha^{*}} \alpha^{2}, \\
& \text { (ii) } g_{21}=\bar{D} \tau_{0} \alpha^{*}\left(2 a l+2 b l W_{11}^{(1)}(0)+b l W_{20}^{(1)}(0)\right),
\end{aligned}
$$

where $W_{11}^{(1)}(0)=\left(-i g_{11} / \omega_{0} \tau_{0}\right) \alpha+\left(i \bar{g}_{11} / \omega_{0} \tau_{0}\right) \bar{\alpha}+E_{2}^{(1)}$, $W_{20}^{(1)}(0)=\left(i g_{20} / \omega_{0} \tau_{0}\right) \alpha+\left(i \overline{g_{02}} / 3 \omega_{0} \tau_{0}\right) \bar{\alpha}+E_{1}^{(1)}, E_{1}^{(1)}=$ $4 l c \alpha^{2} \omega_{0} \tau_{0} i /\left(d+k e^{-i \omega_{0} \tau_{0}}-4 \omega_{0}^{2} \tau_{0}^{2}-2 l c \omega_{0} \tau_{0}\right), E_{2}^{(1)}=2 \alpha \bar{\alpha}$.

Proof. (i) Noticing $x_{t}(\theta)=\left(x_{1 t}(\theta), x_{2 t}(\theta)\right)=W(t, \theta)+z q(\theta)+$ $\bar{z} \overline{q(\theta)}$ and $q(\theta)=(\alpha, \beta)^{T} e^{i \omega_{0} \tau_{0} \theta}$, we have

$$
\begin{aligned}
x_{1 t}(0)= & z+\bar{z}+W_{20}^{(1)}(0) \frac{z^{2}}{2} \\
& +W_{11}^{(1)}(0) z \bar{z}+W_{02}^{(1)}(0) \frac{\bar{z}^{2}}{2}+\cdots
\end{aligned}
$$

By (32), we obtain

$$
\begin{aligned}
g(z, \bar{z}) & =\bar{q}^{*}(0) f_{0}(z, \bar{z}) \\
& =\bar{D} \tau_{0}\left(\overline{\alpha^{*}}, \overline{\beta^{*}}\right)\left(\frac{1}{3} a l x_{1 t}^{3}(0)+\frac{1}{2} b l x_{1 t}^{2}(0), 0\right)^{T} .
\end{aligned}
$$

Substituting (34) into the above equation and comparing the coefficients with (32), we obtain the results.

(ii) The detail procedure of proof refers to [5] and [9-11]. This completes the proof.

According to Proposition 5, we can compute the following parameters:

$$
\begin{gathered}
C_{1}(0)=\frac{i}{2 \tau_{0} \omega_{0}}\left(g_{20} g_{11}-2\left|g_{11}\right|^{2}-\frac{1}{3}\left|g_{02}\right|^{2}\right)+\frac{g_{21}}{2}, \\
\mu_{2}=-\frac{\operatorname{Re}\left\{C_{1}(0)\right\}}{\operatorname{Re}\left\{\lambda^{\prime}\left(\tau_{0}\right)\right\}}, \\
\beta_{2}=2 \operatorname{Re}\left\{C_{1}(0)\right\}, \\
T_{2}=-\frac{\operatorname{Im}\left\{C_{1}(0)\right\}+\mu_{2}\left(\operatorname{Im}\left\{\lambda^{\prime}\left(\tau_{0}\right)\right\}\right)}{\omega_{0}} .
\end{gathered}
$$

From the discussion in Section 2 we know that $\operatorname{Re}\left\{\lambda^{\prime}\left(\tau_{0}\right)\right\}>0$. We therefore have the following result.

Theorem 6. If $\operatorname{Re}\left\{C_{1}(0)\right\}<0$ (>0), the direction of the Hopf bifurcation of the system (1) at the equilibrium $(0,0)$ when $\tau=\tau_{0}$ is supercritical (subcritical) and the bifurcating periodic solutions are orbitally asymptotically stable (unstable).

Finally, we give a concrete example to illustrate the dynamics behaviour of the Raleigh model.

We take the coefficients $d=-7, l=2, c=-2, k=9$ in (3). Omitting these complicated expressions, we obtain the numerical results directly by means of the software MatLab: $\omega_{0} \doteq 0.5871, \tau_{0} \doteq 0.935, \operatorname{Re}\left\{\lambda^{\prime}\left(\tau_{0}\right)\right\} \doteq 2.2695 \times 10^{-5}$. So, we directly compete $C_{1}(0) \doteq-0.0431+0.0381 i, \mu_{2} \doteq 12.4372$, $\beta_{2} \doteq-0.862$. 


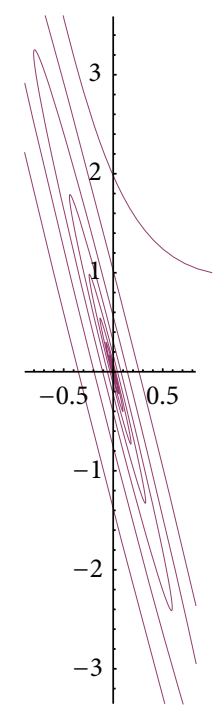

Figure 1: The equilibrium $(0,0)$ of system (3) is stable with $\tau=0.8<$ $\tau_{0}=0.935$.

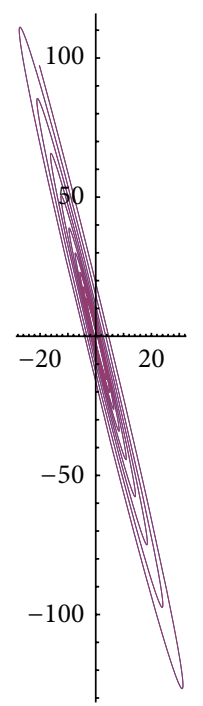

FIgURE 2: The equilibrium $(0,0)$ of system (3) is unstable with $\tau=$ $0.97>\tau_{0}=0.935$.

According to Theorem 6, $\operatorname{Re}\left\{C_{1}(0)\right\} \doteq-0.0431<0$. That is, the bifurcating periodic solutions of system (3) with the above coefficients are supercritical and orbitally asymptotically stable at $\tau=\tau_{0}$.

Thus, the conclusion confirms the effectiveness of our research results.

\section{Conclusion}

Firstly, under the condition of $\tau=0$, we discuss the Reyleigh price model. We know that the stability of price varies with the parameters changing. When $c<0, k+d>0$ and $c=0$, $k+d>0, b<0$, the price tends to the stability. The other cases are unstable. morever, we discuss the Reyleigh price model with time delay (3). By adjusting the parameters $d$,

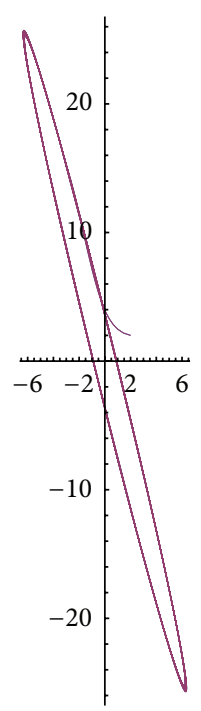

Figure 3: When $\tau_{0}=0.935$, the periodic solutions occur from the equilibrium $(0,0)$.

$k$, we more easily control the price such that the price tends to our expected results. For example, when we take $a=0$, $b=0, d=-7, l=2, c=-2, k=9, \tau=0.8$ in the system (3), the equilibrium $(0,0)$ of system (3) is stable (see Figure 1). By contrast, when we take $a=0, b=0, d=-7$, $l=2, c=-2, k=9, \tau=0.97$ in the system (3), the $(0,0)$ is unstable and there occurs a periodic solution around (0.0) (see Figures 2 and 3). So, by shortening the time delay between the supply and the demand, we can keep the price stable. On the contrary, the price is unstable and undergoes a periodic oscillation. However our analysis indicates that the dynamics of the Reyleigh price model with time delay can be much more complicated than we may have expected. It is still interesting and inspiring to research the price.

\section{References}

[1] T. Lv and Z. Liu, "Hopf bifurcation of price Reyleigh equation with time delay," Journal of Jilin University, vol. 47, no. 3, pp. 441448, 2009.

[2] Z. Xi-fan, C. Xia, and C. Yun-qing, "A qualitative analysis of price model in differential equations of price," Journal of Shenyang Institute of Aeronautical Engineering, vol. 21, no. 1, pp. 83-86, 2004.

[3] H. I. Freedman, J. W.-H. So, and P. Waltman, "Coexistence in a model of competition in the chemostat incorporating discrete delays," SIAM Journal on Applied Mathematics, vol. 49, no. 3, pp. 859-870, 1989.

[4] J. Wei and C. Yu, "Hopf bifurcation analysis in a model of oscillatory gene expression with delay," Proceedings of the Royal Society of Edinburgh A, vol. 139, no. 4, pp. 879-895, 2009.

[5] Y. Song, J. Wei, and M. Han, "Local and global Hopf bifurcation in a delayed hematopoiesis model," International Journal of Bifurcation and Chaos in Applied Sciences and Engineering, vol. 14, no. 11, pp. 3909-3919, 2004.

[6] J. K. Hale and S. M. V. Lunel, Introduction to Functional Differential Equations, Springer, Berlin, Germany, 1995. 
[7] G. Mircea, M. Neamtu, and D. Opris, Dynamical Systems from Economy, Mechanic and Biology Described by Differential Equations with Time Delay, Editura Mirton, Timişoara, Romania, 2003 (Romanian).

[8] H. Bai and Y. Zhai, "Hopf bifurcation analysis for the model of the chemostat with one species of organism," Abstract and Applied Analysis, vol. 2013, Article ID 829045, 7 pages, 2013.

[9] Y. Song and J. Wei, "Bifurcation analysis for Chen's system with delayed feedback and its application to control of chaos," Chaos, Solitons \& Fractals, vol. 22, no. 1, pp. 75-91, 2004.

[10] J. Wei, "Bifurcation analysis in a scalar delay differential equation," Nonlinearity, vol. 20, no. 11, pp. 2483-2498, 2007.

[11] S. Ruan and J. Wei, "On the zeros of transcendental functions with applications to stability of delay differential equations with two delays," Dynamics of Continuous, Discrete \& Impulsive Systems A, vol. 10, no. 6, pp. 863-874, 2003. 


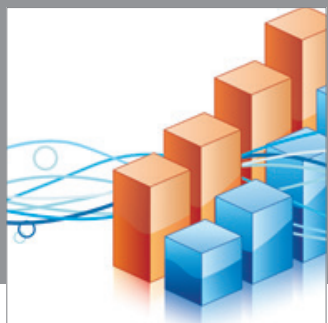

Advances in

Operations Research

mansans

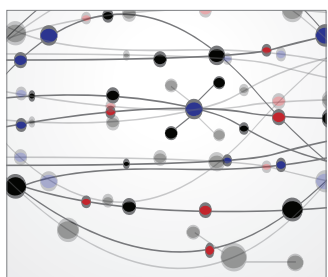

The Scientific World Journal
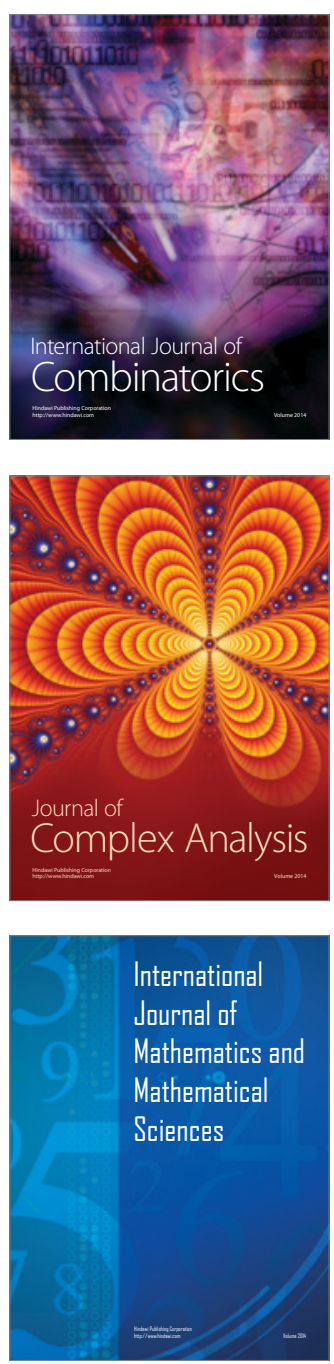
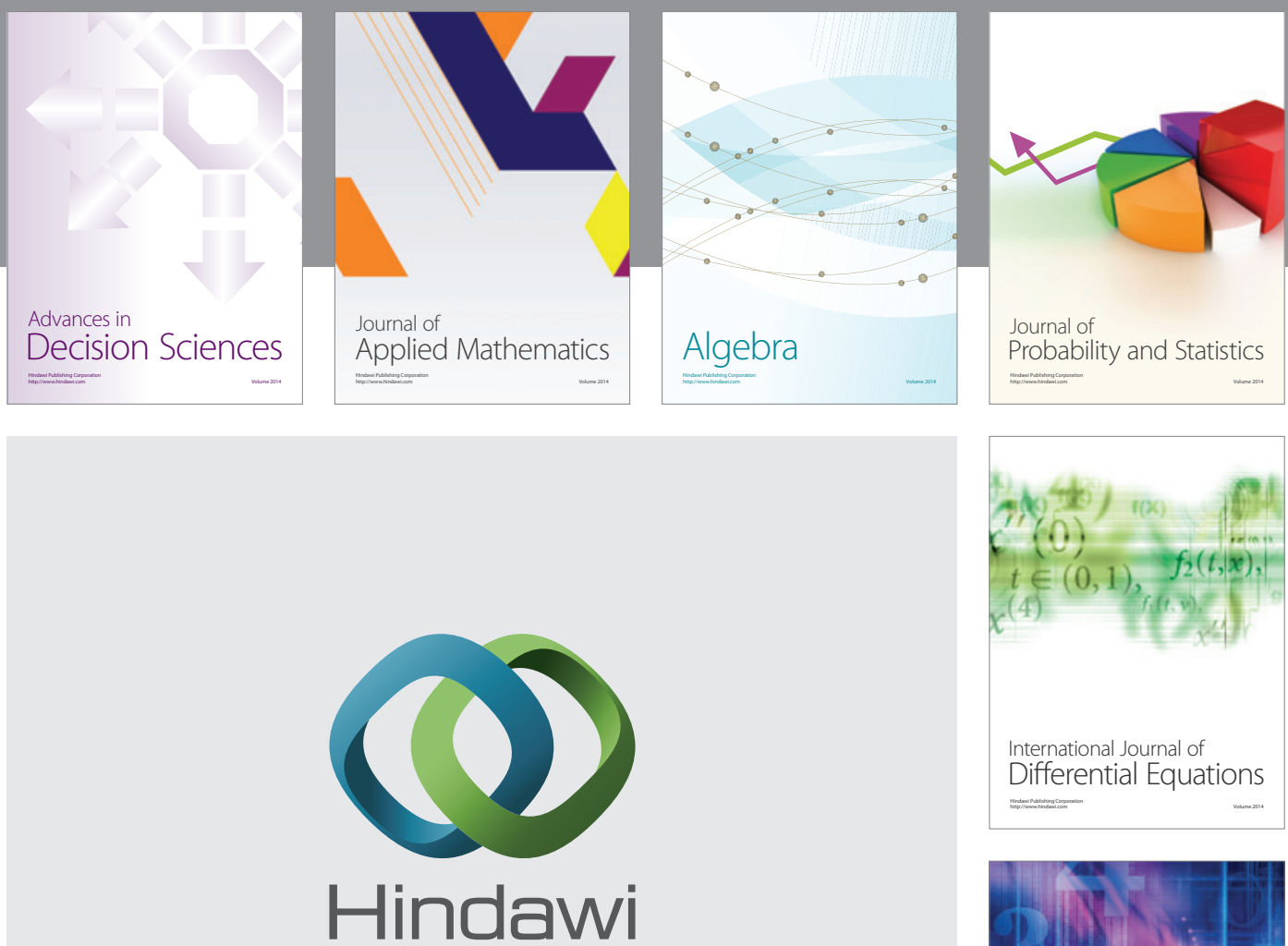

Submit your manuscripts at http://www.hindawi.com
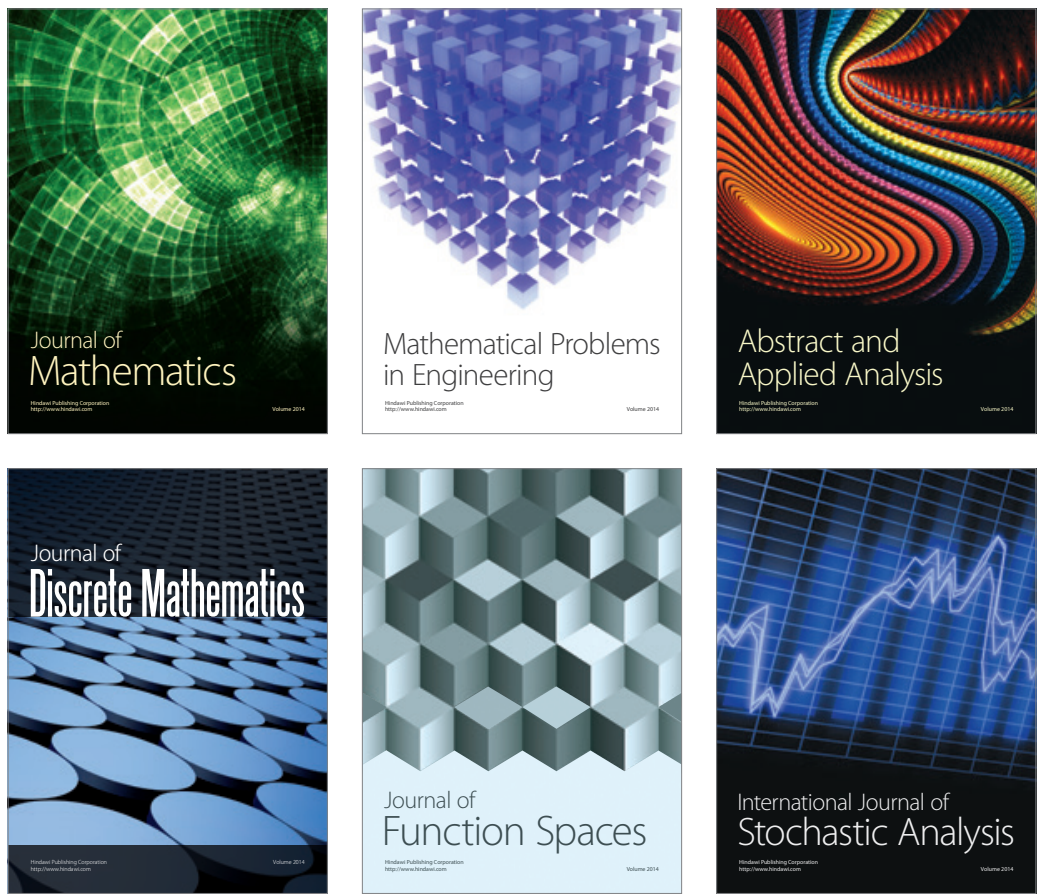

Journal of

Function Spaces

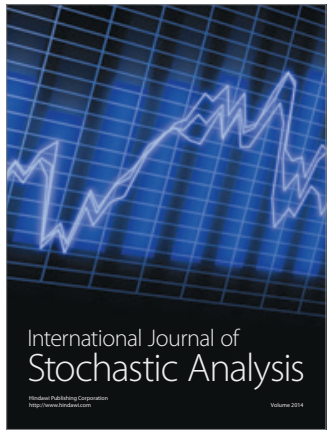

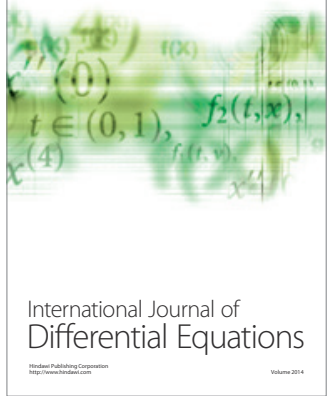
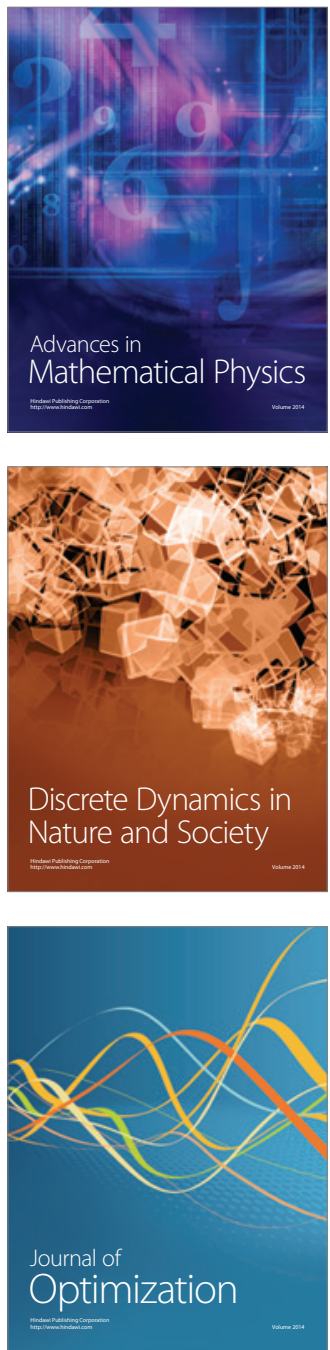